\title{
Interim Results of Post-Marketing Observational Study of Omidenepag Isopropyl for Glaucoma and Ocular Hypertension in Japan
}

\author{
Toru Nakazawa $\cdot$ Kanji Takahashi · Yasuaki Kuwayama • \\ Akio Nomura $\cdot$ Fumiki Shimada
}

Received: November 7, 2021 / Accepted: December 22, 2021 / Published online: January 20, 2022

(c) The Author(s) 2022

\begin{abstract}
Introduction: This post-marketing observational interim analysis evaluated the 12-month effectiveness and safety of omidenepag isopropyl (OMDI) ophthalmic solution in daily clinical settings.

Methods: This was a multicenter, large-scale, non-interventional, prospective, observational study conducted in Japan. The target enrollment was 3900 patients, and the overall observation period was 12 months. Patients with glaucoma and ocular hypertension $(\mathrm{OH})$ with no previous history of OMDI use were enrolled. The key endpoints were change in intraocular pressure (IOP) from baseline and the incidence of adverse reactions (ADRs).
\end{abstract}

T. Nakazawa

Department of Ophthalmology, Tohoku University,

2-1 Seiryo-machi, Aoba-ku, Sendai 980-8575, Japan

K. Takahashi

Department of Ophthalmology, Kansai Medical

University, 2-5-1 Shin-machi, Hirakata, Osaka 573-

1010, Japan

Y. Kuwayama

Fukushima Eye Clinic, 5-6-16, Fukushima,

Fukushima-ku, Osaka 553-0003, Japan

A. Nomura $(\bowtie) \cdot$ F. Shimada

Santen Pharmaceutical Co., Ltd, 4-20, Ofukacho,

Kita-ku, Osaka 530-8552, Japan

e-mail: akio.nomura@santen.com
Results: A total of 1862 patients were evaluated in this 12-month interim analysis. Most patients were diagnosed with normal-tension glaucoma (NTG, 62.0\%). The treatment patterns with OMDI were naïve monotherapy (48.4\%), switching monotherapy (18.4\%), and concomitant therapy $(31.1 \%)$. The overall incidence of ADRs was $24.3 \%$, which was similar between the monotherapy and concomitant therapy groups. Common ADRs were conjunctival hyperemia, refractive disorder, and myopia. Macular edema was observed in four patients. No ADRs categorized as prostaglandinassociated periorbitopathy were observed. There was a significant reduction in mean IOP at 12 months, with a change of $-1.9 \pm 2.9 \mathrm{mmHg}$ from baseline (reduction $-10.4 \pm 16.5 \%$ ). The mean IOP change from baseline was $-2.7 \pm$ $2.6 \mathrm{mmHg}$ in the naïve monotherapy group, $-1.1 \pm 2.6 \mathrm{mmHg}$ in the switching monotherapy group, and $-1.6 \pm 3.1 \mathrm{mmHg}$ in the concomitant therapy group (all $P<0.05$ ). The mean IOP decreased by $-2.5 \pm 3.2 \mathrm{mmHg},-1.5 \pm$ $2.4 \mathrm{mmHg}$, and $-2.3 \pm 4.5 \mathrm{mmHg}$ in the primary open-angle glaucoma (POAG), NTG, and $\mathrm{OH}$ groups, respectively. The treatment persistence with OMDI was $82.4 \%$.

Conclusion: This study demonstrated the safety and efficacy of OMDI for glaucoma and $\mathrm{OH}$ as monotherapy and concomitant therapy in daily clinical settings. In this interim analysis, OMDI showed a favorable benefit-risk profile, and can be first-line therapy for glaucoma. 
Keywords: Adverse reaction; Glaucoma; Intraocular pressure; Omidenepag isopropyl; Ophthalmology; Post-marketing

\section{Key Summary Points}

Why carry out this study?

There is currently a lack of evidence on the use of omidenepag isopropyl with other antiglaucoma agents in patients with glaucoma and ocular hypertension

In this 12-month post-marketing interim analysis, the safety and efficacy of omidenepag isopropyl (OMDI) were evaluated in patients with glaucoma and ocular hypertension $(\mathrm{OH})$ with no previous history of OMDI usage

\section{What was learned from the study?}

In patients who were receiving OMDI as a monotherapy or in combination with other drugs, a greater improvement in intraocular pressure (IOP) and a higher treatment persistence were observed without compromising its safety

After 3 months of treatment, patients treated with OMDI had a significant reduction in IOP from the baseline, and maintained around $14 \mathrm{mmHg}$ stably across all treatment patterns, including naïve, switching, and concomitant usage of OMDI; the reduction in IOP was also consistent in patients diagnosed with normal-tension glaucoma (NTG), primary open-angle glaucoma (POAG), or $\mathrm{OH}$. Treatment with OMDI was not associated with any events of prostaglandinassociated periorbitopathy

This study shows that OMDI can be preferred in treatment-naïve patients or as a concomitant therapy in patients who require switching

\section{INTRODUCTION}

Glaucoma is an optic nerve disorder that can lead to an irreversible vision loss when left untreated [1], with an estimated prevalence of 111.8 million worldwide by 2040 [2]. Lowering the intraocular pressure (IOP) is the only evidence-based treatment strategy to delay the progression of glaucoma $[1,2]$. Glaucoma can be divided into open-angle glaucoma (OAG) and angle-closure glaucoma (ACG) [2]. Primary open-angle glaucoma (POAG) is the most common type of glaucoma, accounting for approximately three-quarters of all cases [3-5]. Normal-tension glaucoma (NTG) is a kind of POAG in which IOP is within the normal range when diagnosed. In ocular hypertension $(\mathrm{OH})$, there are no signs of optic nerve damage, but the IOP is elevated. Evidence based on large randomized controlled trials $[6,7]$ indicate that treatment with topical ocular hypotensive agents reduces the elevated IOP and delays or prevents disease progression of OAG.

Available topical hypotensive agents, such as $\beta$-blockers, carbonic dehydratase inhibitors (CAI), and alpha-adrenergic agonists, reduce the aqueous humor outflow, while prostaglandin $\mathrm{F}_{2 \alpha}$ analogs (PGAs), rho-associated protein kinase (ROCK) inhibitors, and parasympathomimetics increase the aqueous humor outflow.

Prostaglandins are the recommended firstline therapy and are the most frequently prescribed medication for glaucoma [8-12]. The target IOP in progressing glaucoma can often be difficult to maintain, as it requires multiple drugs. Patients with low/non-response to PGA are recommended to receive adjunctive therapy or are switched to an alternative agent $[11,13]$. Moreover, in a previous study, $36 \%$ of 16,486 patients initially treated with PGAs required an adjunctive IOP-lowering therapy within 24 months [13]. In addition, PGAs are known to show prostaglandin-associated periorbitopathy (PAP), including eyelid or iris pigmentation, elongated or darkened eyelashes, and deepening upper eyelid sulcus [14]. Thus, new pharmacologic agents with new mechanisms of action are needed to expand the available 
treatment regimens to maintain the IOP of patients with POAG or $\mathrm{OH}$.

Omidenepag isopropyl (OMDI) is a prodrug of a pharmacologically active acid metabolite, omidenepag (UR-7276), a selective, prostanoid EP2 receptor agonist (EP2 agonist) [15-17]. OMDI reduces the IOP by binding to an EP2 receptor, thereby increasing the aqueous humor outflow via the trabecular and uveoscleral pathways [17]. It was initially approved in Japan in 2018 as Eybelis ${ }^{\circledR}$ ophthalmic solution $0.002 \%$ for the treatment of glaucoma and $\mathrm{OH}$, and it was later approved in Korea, Taiwan, India, Thailand, Singapore, and Malaysia [18].

In previous clinical trials, OMDI monotherapy was non-inferior to latanoprost and showed a significant IOP-lowering effect in latanoprost non/low-responders [19, 20]; also, the combination of OMDI with other glaucoma medications effectively lowered the IOP [21]. A recent 1-year phase 3 study demonstrated a continuous reduction in IOP with OMDI monotherapy or with concomitant administration with timolol [22].

While OMDI has been studied in multiple clinical trials, there is a limited information on its concomitant use with other antiglaucoma drugs besides tafluprost and $\beta$-blockers [22]. The incidence of adverse drug reactions (ADRs), such as ocular inflammation, corneal thickening, and macular edema, in real-world settings also remains unclear. Hence, this large, postmarketing, observational study was conducted to assess the effectiveness and safety of OMDI as a monotherapy and in combination with other antiglaucoma drugs, and this report presents a result of interim analysis. This evidence in daily clinical settings in Japan is expected to contribute to other countries as reference data for their clinical settings.

\section{METHODS}

\section{Study Design and organization}

This multicenter, large-scale, post-marketing, non-interventional, prospective, observational study was conducted in Japan in accordance with the Good Post-Marketing Study Practice
(MHLW Ordinance No. 171; December 20, 2004). The subject enrollment period was from December 21, 2018, to June 30, 2023. The entire study was scheduled for 7 years (November 27, 2018 , to November 30, 2025), and is ongoing. The current interim analysis includes the data from patients retrieved until March 2021. The study was sponsored by Santen Pharmaceutical, the marketing authorization holder of OMDI in Japan. The study protocol was reviewed and approved by regulatory authorities; hence, an additional ethics review board approval was not mandated.

\section{Patients and Registration}

The target enrollment was 3900 patients. Patients with glaucoma and $\mathrm{OH}$ with no previous history of OMDI use were enrolled. The key exclusion criteria were patients with aphakic or pseudophakic eyes, and patients receiving tafluprost as a concomitant drug with OMDI, which was contraindicated. Patient enrollment was conducted using a central registration system to eliminate selection bias. All patients provided an oral or written informed consent. At the time of patient registration, the patients were classified under the monotherapy group or the concomitant therapy group, depending on whether or not other antiglaucoma drugs were used along with OMDI. Patients who started receiving OMDI as a monotherapy, but also started receiving other antiglaucoma/OH drugs during the study, were classified under the intermediate concomitant group. The data in the intermediate concomitant group were completely excluded from both monotherapy and concomitant therapy groups. For evaluation, monotherapy patients were further classified under the naïve monotherapy group when they never received any antiglaucoma therapy before starting OMDI and the switching monotherapy group when the current antiglaucoma treatment was switched to OMDI.

\section{Data Collection and Analysis}

The observation period was 12 months after starting the administration of OMDI in both 
monotherapy and concomitant therapy patients. The data were collected using electronic case report forms (CRFs), which were submitted at 3 months, 7 months, and 12 months after its administration. The following data were non-interventionally collected from medical institutions: patient background, OMDI treatment status, concomitant medications used, IOP, corrected visual acuity, refraction (using spherical refraction), central corneal thickness; results from macular optical coherence tomography (OCT) examination, visual field test, objective assessment of conjunctival hyperemia, corneal fluorescein staining; and ADRs that occurred during OMDI administration. Each objective parameter was evaluated using a four-point score (0: none; 1 : mild; 2 : moderate; 3: severe).

An adverse event (AE) was defined as any untoward medical occurrences in a patient treated with OMDI, regardless of the causal relationship. If a doctor could not rule out the causality between $\mathrm{AE}$ and OMDI, the event was treated as an ADR. The aggregation of ADRs was performed using the MedDRA preferred terms. The objective findings and effectiveness analyses were based on the administration status of the evaluated eye. Only eyes with glaucoma or $\mathrm{OH}$ were assessed. If both eyes could be considered, the eye with a higher IOP value at the start of administration was assessed, and if the IOP of the left and right eyes was the same, the right eye was assessed. Patient-based tabulation was performed regardless of the evaluated eye.

The following fluctuations in ophthalmologic examination parameters (hereafter, parameter fluctuations) were noted regardless of each doctors' assessment: refractivity fluctuation, decrease or increase in refraction by $\geq 0.75$ D compared to a previously measured value; central corneal thickness fluctuation, decrease or increase by $\geq 100 \mu \mathrm{m}$ compared to a previously measured value; visual acuity fluctuation, previous deterioration of three or more lines of best-corrected visual acuity in Landolt $\mathrm{C}$ eye chart; IOP fluctuation, an increase by $\geq 50 \%$ compared to a previous measurement and the increased IOP was $\geq 20 \mathrm{mmHg}$; and visual field fluctuation, a decrease by $\geq-6 \mathrm{~dB}$ compared to a previously measured value.
The incidence of parameter fluctuations was included as an ADR; however, incidence tables were separated from Table 4 (see below), which contains the ADRs reported by doctors.

\section{Statistical Analyses}

Data aggregation and analyses were performed using SAS v.9.4 software (SAS Institute, Japan). Descriptive statistics [mean \pm standard deviation (SD)] were used to summarize patient background data. When comparing the IOP between the baseline and that after 3 months, the IOP data were carried over when the 3 -month data were missing (i.e., 1-month or 2-month data served as the 3-month data). Similarly, the 4- or 5-month data served for missing 6-month data, 7-, or 8-month data served for missing 9-month data, and 10- or 11-month data served for missing 12-month data. Paired $t$ tests were used to analyze the IOP, central corneal thickness, and corrected visual acuity, with a two-tailed significance level of $5 \%$. Wilcoxon's signed rank-sum test was used for conjunctival hyperemia and corneal fluorescein staining. The Kaplan-Meier method was used to examine the treatment persistent rate [95\% confidence interval (CI)]. The discontinuation of OMDI due to the occurrence of AEs and insufficient therapeutic effect was defined as a treatment failure.

\section{RESULTS}

The CRFs of 1929 patients were collected from 272 ophthalmology facilities by March 2021 (Fig. 1). Among these, 67 patients (3.5\%) were excluded from the analysis due to the following reasons: absence of post-treatment data due to no revisit (53 patients), registration criteria violation (11 patients), and unconfirmable existence of $\mathrm{AE}$ (3 patients). For the current interim analysis, 1862 patients were analyzed. CRFs from up to 3,7 , and 12 months were available in 1862, 1233, and 710 patients, respectively. 


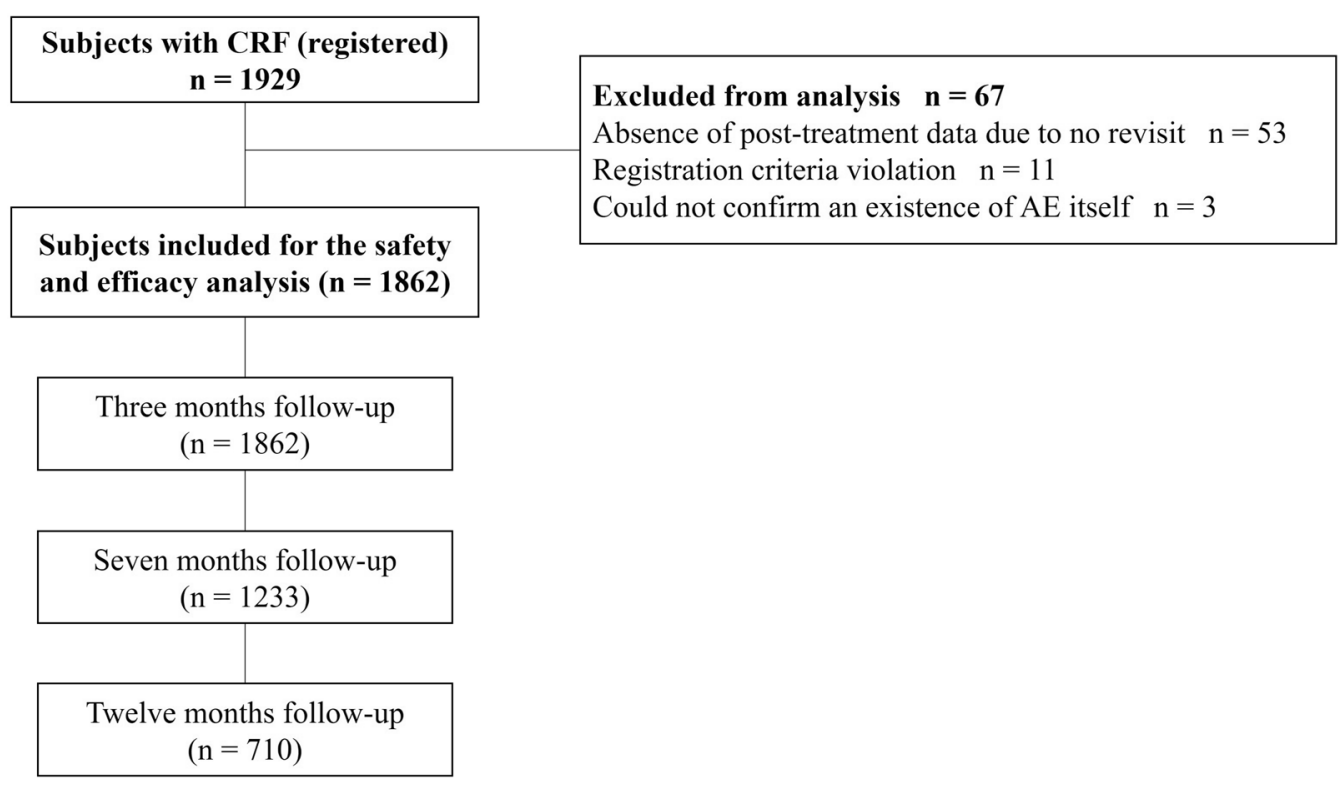

Fig. 1 Patient enrollment and assessment flow diagram

\section{Patient Demographics}

The demographic and clinical characteristics of the study population are presented in Table 1 . This study enrolled 1151 females (61.8\%) and 711 males $(38.2 \%)$, and the mean $( \pm S D)$ age was $59.9 \pm 13.0$ years. Most of the study patients were diagnosed with NTG (1154 patients, $62.0 \%$ ), followed by POAG (558 patients, $30.0 \%)$, PACG (30 patients, $1.6 \%), \mathrm{OH}(87$ patients, $4.7 \%$ ), and others (33 patients, $1.8 \%$ ). The mean IOP at baseline was $16.6 \pm 4.5 \mathrm{mmHg}$. The baseline characteristics were similar between the groups except for the higher ratio of female patients $(70.2 \%)$ in the switching monotherapy group than that in the naïve monotherapy $(58.5 \%)$ and concomitant therapy groups $(61.7 \%)$, and the higher mean Humphrey visual field MD $(-6.6 \mathrm{~dB})$ and patient ratio of $<-12 \mathrm{~dB}(21.3 \%)$ in the concomitant therapy group.

\section{Therapeutic Patterns}

A total of 902 patients $(48.4 \%)$ received OMDI as a naïve monotherapy, 342 patients (18.4\%) switched to OMDI monotherapy from a prior treatment (switching monotherapy), 579 patients $(31.1 \%)$ received OMDI concomitantly, and 39 patients $(2.1 \%)$ received an intermediate concomitant therapy (Table 1). In the switching monotherapy group (342 patients), most patients received PGA $(60.2 \%)$ before starting OMDI, followed by $\beta$-blockers (36.3\%), CAIs (10.5\%), $\alpha 2$ agonists (5.8\%), and ROCK inhibitors $(1.5 \%)$ (Table 2$)$. In the concomitant therapy group $(n=579)$, most patients received $\beta$ blockers $(74.4 \%)$, followed by CAIs $(56.1 \%)$; only a few patients received PGAs (6.6\%; Table 3). In total patients, the timing of receiving OMDI was at night in $85.5 \%$ and in the morning in $13.8 \%$.

\section{Safety}

The overall incidence of ADRs was 24.3\% (452/ 1862 patients). There was no significant difference of ADR incidence between the naïve monotherapy group (23.2\%), switching monotherapy group (25.2\%), and concomitant therapy group (25.6\%). The most frequently reported ADRs were conjunctival hyperemia $(3.6 \%)$, refractive disorders $(3.1 \%)$, and myopia (1.1\%) (Table 4). Four serious ADRs were 
Table 1 Patient demographics and baseline characteristics $(\mathrm{n}=1862)$

\begin{tabular}{|c|c|c|c|c|}
\hline Characteristics & $\begin{array}{l}\text { Overall } \\
n=1862 \\
n(\%)\end{array}$ & $\begin{array}{l}\text { Naïve } \\
\text { monotherapy } \\
n=902 \\
n(\%)\end{array}$ & $\begin{array}{l}\text { Switching } \\
\text { monotherapy } \\
n=342 \\
n(\%)\end{array}$ & $\begin{array}{l}\text { Concomitant } \\
\text { therapy } \\
n=579 \\
n(\%)\end{array}$ \\
\hline \multicolumn{5}{|l|}{ Sex } \\
\hline Male & $711(38.2)$ & $374(41.5)$ & $102(29.8)$ & $222(38.3)$ \\
\hline Female & $1151(61.8)$ & $528(58.5)$ & $240(70.2)$ & $357(61.7)$ \\
\hline \multicolumn{5}{|l|}{ Mean age [years] } \\
\hline Mean \pm SD & $59.9 \pm 13.0$ & $58.8 \pm 12.8$ & $59.8 \pm 13.2$ & $61.8 \pm 12.9$ \\
\hline$<65$ & $1125(60.4)$ & $579(64.2)$ & $210(61.4)$ & $311(53.7)$ \\
\hline$\geq 65$ & $737(39.6)$ & $323(35.8)$ & $132(38.6)$ & $268(46.3)$ \\
\hline \multicolumn{5}{|l|}{ Diagnosis } \\
\hline POAG & $558(30.0)$ & $235(26.1)$ & $94(27.5)$ & $220(38.0)$ \\
\hline NTG & $1154(62.0)$ & $621(68.8)$ & $211(61.7)$ & $301(52.0)$ \\
\hline PACG & $30(1.6)$ & $8(0.9)$ & $4(1.2)$ & $14(2.4)$ \\
\hline Other types of glaucoma & $33(1.8)$ & $7(0.8)$ & $4(1.2)$ & $18(3.1)$ \\
\hline $\mathrm{OH}$ & $87(4.7)$ & $31(3.4)$ & $29(8.5)$ & $26(4.5)$ \\
\hline \multicolumn{5}{|l|}{ Treatment pattern } \\
\hline Naïve monotherapy & $902(48.4)$ & & & \\
\hline Switching monotherapy & $342(18.4)$ & - & - & - \\
\hline Concomitant therapy & $579(31.1)$ & & & \\
\hline Intermediate concomitant & $39(2.1)$ & & & \\
\hline \multicolumn{5}{|l|}{ Ocular complications } \\
\hline No & $1031(55.4)$ & $529(58.6)$ & $157(45.9)$ & $325(56.1)$ \\
\hline Yes & $831(44.6)$ & $373(41.4)$ & $185(54.1)$ & $254(43.9)$ \\
\hline Retinal/vitreous disease & $121(6.5)$ & $54(6.0)$ & $27(7.9)$ & $37(6.4)$ \\
\hline Cataract/lens disease & $360(19.3)$ & $177(19.6)$ & $72(21.1)$ & $107(18.5)$ \\
\hline Iris, ciliary body, choroidal disease & $10(0.5)$ & $2(0.2)$ & $5(1.5)$ & $3(0.5)$ \\
\hline Corneal and conjunctival disease & $455(24.4)$ & $193(21.4)$ & $102(29.8)$ & $149(25.7)$ \\
\hline Eyelid and lacrimal disorders & $37(2.0)$ & $10(1.1)$ & $11(3.2)$ & $16(2.8)$ \\
\hline
\end{tabular}


Table 1 continued

\begin{tabular}{|c|c|c|c|c|}
\hline Characteristics & $\begin{array}{l}\text { Overall } \\
n=1862 \\
n(\%)\end{array}$ & $\begin{array}{l}\text { Naïve } \\
\text { monotherapy } \\
n=902 \\
n(\%)\end{array}$ & $\begin{array}{l}\text { Switching } \\
\text { monotherapy } \\
n=342 \\
n(\%)\end{array}$ & $\begin{array}{l}\text { Concomitant } \\
\text { therapy } \\
n=579 \\
n(\%)\end{array}$ \\
\hline \multicolumn{5}{|l|}{ Humphrey visual field MD $[\mathrm{dB}]$} \\
\hline Mean $\pm S D$ & $-4.4 \pm 5.5$ & $-3.7 \pm 4.6$ & $-2.8 \pm 3.6$ & $-6.6 \pm 7.0$ \\
\hline$>-6$ & $572(75.6)$ & $313(80.5)$ & $113(86.3)$ & $134(60.6)$ \\
\hline-6 to -12 & $109(14.4)$ & $52(13.4)$ & $14(10.7)$ & $40(18.1)$ \\
\hline$<-12$ & $76(10.0)$ & $24(6.2)$ & $4(3.1)$ & $47(21.3)$ \\
\hline Not measured & 1105 & 513 & 211 & 358 \\
\hline $\begin{array}{l}\text { Intraocular pressure, mean } \pm S D \\
\quad[\mathrm{mmHg}]\end{array}$ & $16.6 \pm 4.5$ & $17.0 \pm 3.8$ & $15.6 \pm 3.6$ & $16.4 \pm 5.8$ \\
\hline
\end{tabular}

Data are expressed as $n(\%)$, unless specified otherwise

MD mean deviation, NTG normal-tension glaucoma, OH ocular hypertension, PACG primary angle-closure glaucoma, POAG primary open-angle glaucoma, SD standard deviation

reported in three patients: two cases had increased IOP, one had an iris synechiae, and one had a macular hole, and their causal relationships with OMDI were not ruled out. Currently, these ADR's outcomes were "improving". There were no observed ADRs categorized as PAP, such as eyelid pigmentation, eyelash abnormality, and deepening of the upper eyelid sulcus. In parameter fluctuations which were noted in regardless of each doctors' assessment, refractivity fluctuation (8.4\%), and visual acuity fluctuation $(2.0 \%)$ were the major fluctuations in all patients. There was no difference in parameter fluctuations between the monotherapy and concomitant therapy groups (Table 5). A total of 4 cases of macular edema including cystoid macular edema $(0.2 \%)$ were observed; all had phakic eyes on both sides. These patients were aged 59, 60, 64 years and in her 60s; 3 were females. The onset of macular edema was 22,31 52 , and 190 days from the start of the OMDI treatment. In all patients, the condition was reported to be recovered or improved 31-111 days after the discontinuation of OMDI. In one patient, the shape of macular edema was reported as a cyst in inner retina without retinal detachment. It was considered to be moderate case. We could not identify any specific common background for these 4 patients. One patient had an epiretinal membrane and dry eye as concomitant ocular diseases. One patient had a concomitant lattice retinal degeneration and a history of retinal photocoagulation. One patient had a cataract, dry eye, and a history of trabeculectomy. The remaining patient had no concomitant disease or any disease history.

A conjunctival hyperemia score of $\geq 1$ was observed in $9.5 \%$ of patients at baseline $(n=1774), 16.6 \%$ at 3 months and $8.3 \%$ at 12 months (Fig. 2). The mean conjunctival hyperemia score was $0.1 \pm 0.4$ at baseline, $0.2 \pm 0.4$ at 3 months, $0.1 \pm 0.3$ at 6 months, and $0.1 \pm 0.3$ at 123 months with statistical significance at 3 months $(p<0.05)$, but not at 6 or 12 months.

The mean corneal staining score was $0.1 \pm 0.4$ at baseline $(n=1436)$. There was no statistically significant difference in scores versus baseline at any time points.

\section{Changes in Ophthalmologic Parameters}

No statistically significant changes were observed in visual acuity (log value) score 
Table 2 Antiglaucoma therapy in patients before switching to OMDI monotherapy

\begin{tabular}{lc}
\hline Component & $\begin{array}{c}\text { Patients switched from } \\
\text { other therapy }(\boldsymbol{n}=\mathbf{3 4 2})\end{array}$ \\
\hline PGA & $206(60.2 \%)$ \\
$\beta$-blocker & $124(36.3 \%)$ \\
CAI & $36(10.5 \%)$ \\
$\alpha_{2}$ agonist & $20(5.8 \%)$ \\
ROCK inhibitor & $5(1.5 \%)$ \\
\hline
\end{tabular}

Multiple entries

CAI carbonic anhydrase inhibitors, OMDI omidenepag isopropyl, PGA prostaglandin, ROCK rho-associated protein kinase

Table 3 Concomitant ocular hypotensive agents in the concomitant therapy group

\begin{tabular}{ll}
\hline Component & Concomitant therapy $(\boldsymbol{n}=\mathbf{5 7 9})$ \\
\hline$\beta$-blocker & $431(74.4 \%)$ \\
CAI & $325(56.1 \%)$ \\
$\alpha_{2}$ agonist & $167(28.8 \%)$ \\
ROCK inhibitors & $64(11.1 \%)$ \\
PGA & $38(6.6 \%)$ \\
\hline
\end{tabular}

Multiple entries

CAI carbonic anhydrase inhibitors, PGA prostaglandin, ROCK rho-associated protein kinase

$[0.022 \pm 0.136$ at baseline $(n=1660)$ to $0.018 \pm 0.123$ at 12 months $(n=216)]$.

The mean central corneal thickness was $539.8 \pm 35.6 \mu \mathrm{m}$ at baseline $(n=153)$ and $544.6 \pm 34.9 \mu \mathrm{m}$ at 12 months $(n=15)$, as measured by a pachymeter, and was $523.8 \pm 42.3 \mu \mathrm{m}$ at baseline $(n=222)$ and $535.4 \pm 41.2 \mu \mathrm{m}$ at 12 months $(n=22)$, as measured by specular microscopy. No statistically significant changes in refraction were observed between the baseline and at any time points.

\section{Efficacy}

The mean IOP for all patients was $16.6 \pm 4.5 \mathrm{mmHg}(n=1836)$ at baseline. The mean IOP decreased significantly $(p<0.05)$ and was maintained around $14 \mathrm{mmHg}$ stably throughout the study period. The mean change in IOP at 12 months was $-1.9 \pm 2.9 \mathrm{mmHg}$, with $-10.4 \pm 16.5 \%$ change from baseline (Fig. 3).

The mean IOP at baseline in the naïve monotherapy, switching monotherapy, and concomitant therapy groups were $17.0 \pm$ $3.8 \mathrm{mmHg}, \quad 15.6 \pm 3.7 \mathrm{mmHg}$, and $16.4 \pm 5.8$ $\mathrm{mmHg}$, respectively. The mean IOP decreased significantly over 12 months, regardless of these treatment patterns $(p<0.05)$. The mean IOP change from the baseline to 12 months was $-2.7 \pm 2.6 \mathrm{mmHg} \quad(-15.1 \% \pm 13.7 \%) \quad$ in the naïve monotherapy group, $-1.1 \pm 2.6$ $\mathrm{mmHg}(-4.9 \% \pm 16.2 \%)$ in the switching monotherapy group, and $-1.6 \pm 3.1 \mathrm{mmHg}$ $(-8.5 \% \pm 17.6 \%)$ in the concomitant therapy group (Fig. 4).

The mean baseline IOP of patients with POAG, NTG, and $\mathrm{OH}$ was $18.4 \pm 5.0 \mathrm{mmHg}$, $15.1 \pm 2.9 \mathrm{mmHg}$, and $22.2 \pm 4.5 \mathrm{mmHg}$, respectively. In the 12-month observation period, the mean IOP of patients with POAG, NTG, and $\mathrm{OH}$ decreased by $-2.5 \pm 3.2 \mathrm{mmHg}$ $(-12.6 \% \pm 17.4 \%),-1.5 \pm 2.4 \mathrm{mmHg}(-9.0 \%$ $\pm 15.3 \%), \quad$ and $-2.3 \pm 4.5 \mathrm{mmHg} \quad(-9.5 \% \pm$ $20.8 \%)$, respectively. The differences were statistically significant at all time points irrespective of the diagnosis $(p<0.05)$ (Fig. 5).

\section{Treatment Persistence}

The overall persistence rate $(95 \% \mathrm{CI})$ over the 12-month observation period was $82.4 \%$ $(79.9-84.7 \%)$ (Fig. 6). The main reasons for treatment discontinuation were the occurrence of AEs $(n=129 ; 35.3 \%)$ and perceived insufficient therapeutic effect $(n=73 ; 20.0 \%)$. 
Table 4 Incidence of ADRs

\begin{tabular}{|c|c|c|c|c|}
\hline Adverse drug reaction (ADR) & $\begin{array}{l}\text { Overall } \\
n=1862 \\
n(\%)\end{array}$ & $\begin{array}{l}\text { Naïve } \\
\text { monotherapy } \\
n=902 \\
n(\%)\end{array}$ & $\begin{array}{l}\text { Switching } \\
\text { monotherapy } \\
n=342 \\
n(\%)\end{array}$ & $\begin{array}{l}\text { Concomitant } \\
\text { therapy } \\
n=579 \\
n(\%)\end{array}$ \\
\hline All ADRs & $452(24.3 \%)$ & $209(23.2 \%)$ & $86(25.2 \%)$ & $148(25.6 \%)$ \\
\hline Conjunctival hyperemia & $67(3.6 \%)$ & $32(3.5 \%)$ & $15(4.4 \%)$ & $19(3.3 \%)$ \\
\hline Refractive disorder & $57(3.1 \%)$ & $25(2.8 \%)$ & $13(3.8 \%)$ & $18(3.1 \%)$ \\
\hline Myopia & $21(1.1 \%)$ & $12(1.3 \%)$ & $5(1.5 \%)$ & $4(0.7 \%)$ \\
\hline Vision blurred & $19(1.0 \%)$ & $9(1.0 \%)$ & $7(2.1 \%)$ & $2(0.4 \%)$ \\
\hline Visual acuity reduced & $19(1.0 \%)$ & $10(1.1 \%)$ & $4(1.2 \%)$ & $5(0.9 \%)$ \\
\hline Intraocular pressure increased & $17(0.9 \%)$ & $4(0.4 \%)$ & $4(1.2 \%)$ & $7(1.2 \%)$ \\
\hline Punctate keratitis & $13(0.7 \%)$ & $7(0.8 \%)$ & $1(0.3 \%)$ & $4(0.7 \%)$ \\
\hline Dry eye & $13(0.7 \%)$ & $10(1.1 \%)$ & - & $3(0.5 \%)$ \\
\hline Iritis & $12(0.6 \%)$ & $5(0.6 \%)$ & $4(1.2 \%)$ & $3(0.5 \%)$ \\
\hline Conjunctivitis allergic & $11(0.6 \%)$ & $7(0.8 \%)$ & $2(0.6 \%)$ & - \\
\hline Eye pain & $9(0.5 \%)$ & $5(0.6 \%)$ & $1(0.3 \%)$ & $3(0.5 \%)$ \\
\hline Visual impairment & $8(0.4 \%)$ & $2(0.2 \%)$ & $3(0.9 \%)$ & $3(0.5 \%)$ \\
\hline Anterior chamber cell & $8(0.4 \%)$ & $4(0.4 \%)$ & $1(0.3 \%)$ & $3(0.5 \%)$ \\
\hline Asthenopia & $7(0.4 \%)$ & $4(0.4 \%)$ & $2(0.6 \%)$ & $1(0.2 \%)$ \\
\hline Photophobia & $6(0.3 \%)$ & $4(0.4 \%)$ & $1(0.3 \%)$ & $1(0.2 \%)$ \\
\hline Abnormal sensation in eye & $6(0.3 \%)$ & $4(0.4 \%)$ & $1(0.3 \%)$ & $1(0.2 \%)$ \\
\hline Foreign body sensation in eyes & $5(0.3 \%)$ & $2(0.2 \%)$ & $3(0.9 \%)$ & - \\
\hline Conjunctivitis & $5(0.3 \%)$ & $2(0.2 \%)$ & $1(0.3 \%)$ & $2(0.4 \%)$ \\
\hline Dizziness & $5(0.3 \%)$ & $3(0.3 \%)$ & $1(0.3 \%)$ & $1(0.2 \%)$ \\
\hline Corneal thickening & $4(0.2 \%)$ & $4(0.4 \%)$ & - & - \\
\hline Eye pruritus & $4(0.2 \%)$ & $2(0.2 \%)$ & $1(0.3 \%)$ & $1(0.2 \%)$ \\
\hline Visual field defect & $4(0.2 \%)$ & $1(0.1 \%)$ & $1(0.3 \%)$ & $2(0.4 \%)$ \\
\hline $\begin{array}{l}\text { Macular edema including cystoid macular } \\
\text { edema }\end{array}$ & $4(0.2 \%)$ & $1(0.1 \%)$ & - & $3(0.5 \%)$ \\
\hline Hyperopia & $3(0.2 \%)$ & $2(0.2 \%)$ & - & $1(0.2 \%)$ \\
\hline
\end{tabular}

ADRs report by $\geq 0.2 \%$ in overall

ADR adverse drug reaction 
Table 5 Parameter fluctuations

\begin{tabular}{lclll}
\hline Parameters & $\begin{array}{l}\text { Overall } \\
\boldsymbol{n}=\mathbf{1 8 6 2} \\
\boldsymbol{n}(\%)\end{array}$ & $\begin{array}{l}\text { Naive monotherapy } \\
\boldsymbol{n}=\mathbf{9 0 2} \\
\boldsymbol{n}(\%)\end{array}$ & $\begin{array}{l}\text { Switching monotherapy } \\
\boldsymbol{n}=\mathbf{3 4 2} \\
\boldsymbol{n}(\%)\end{array}$ & $\begin{array}{l}\text { Concomitant therapy } \\
\boldsymbol{n}=\mathbf{5 7 9} \\
\boldsymbol{n}(\%)\end{array}$ \\
\hline Refractivity fluctuation & $157(8.4 \%)$ & $70(7.8 \%)$ & $26(7.6 \%)$ & $58(10.0 \%)$ \\
Visual acuity fluctuation & $38(2.0 \%)$ & $21(2.3 \%)$ & $5(1.5 \%)$ & $12(2.1 \%)$ \\
Visual field fluctuation & $8(0.4 \%)$ & $1(0.1 \%)$ & - & $7(1.2 \%)$ \\
IOP fluctuation & $5(0.3 \%)$ & $1(0.1 \%)$ & - & $4(0.7 \%)$ \\
\hline
\end{tabular}

IOP intraocular pressure

\section{DISCUSSION}

This interim study reports the safety and effectiveness of OMDI as a naïve monotherapy, switching monotherapy, and in concomitant therapy with other antiglaucoma/OH therapy in 1862 Japanese patients. To the best of our knowledge, this is the largest study on OMDI since its approval in Japan in November 2018.

In terms of efficacy, an overall mean IOP reduction of about 10\% from the baseline was observed throughout the study period. The reduced IOP also remained stable at around $14 \mathrm{mmHg}$ throughout the observation period. In the Japanese population, a similar situation was reported that the IOP was maintained to be $14.3 \pm 3.3 \mathrm{mmHg}$ at 12 months through the dosing start of preservative free travoprost to the patients whose IOP was $14.9 \pm 3.4 \mathrm{mmHg}$ at baseline which had been kept by latanoprost [23]. The significant and stable IOP reduction was observed similarly in the long-term clinical trials [22]. This change in IOP was observed in all the treatment patterns. Among the patients

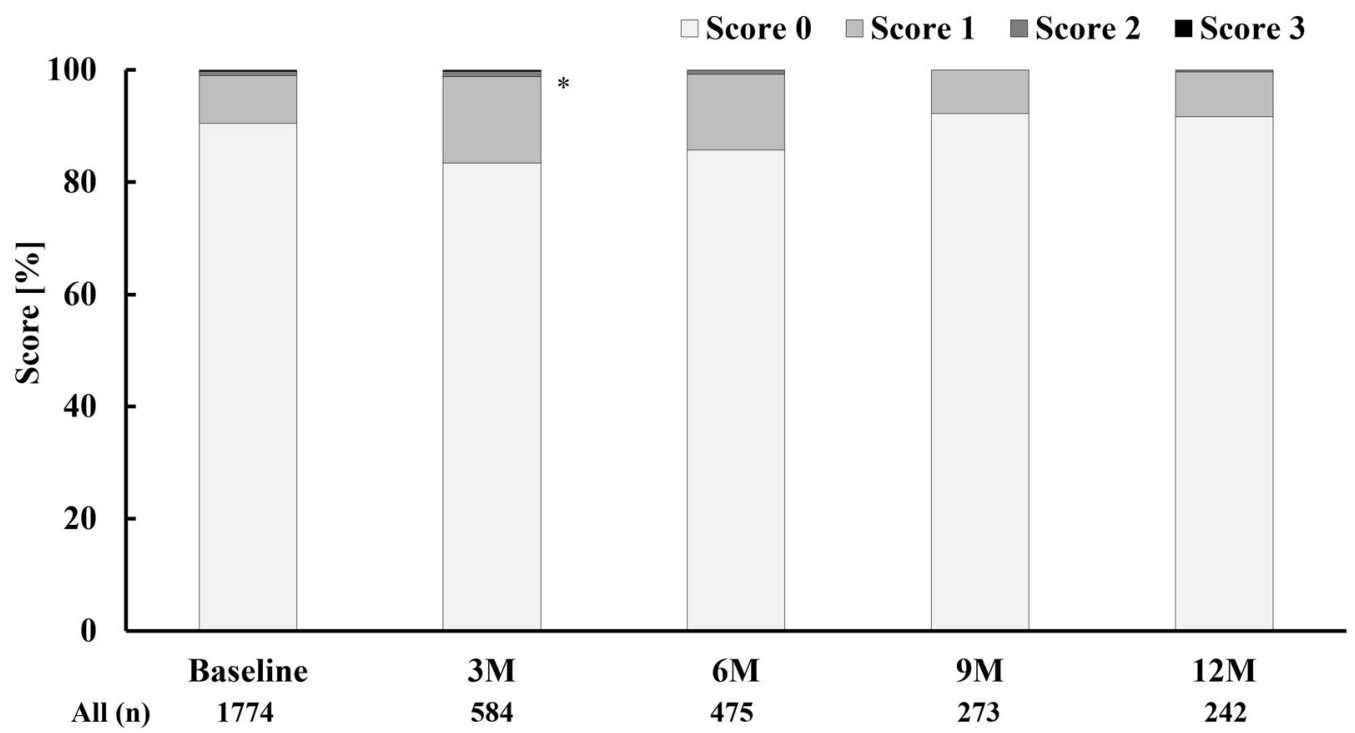

Fig. 2 Percentage of patients for each hyperemia score. The mean change in score for 3 months compared to the baseline score was significant $\left({ }^{*} p<0.05\right.$; Wilcoxon's signed rank-sum test). $n$ number of patients, $M$ months 


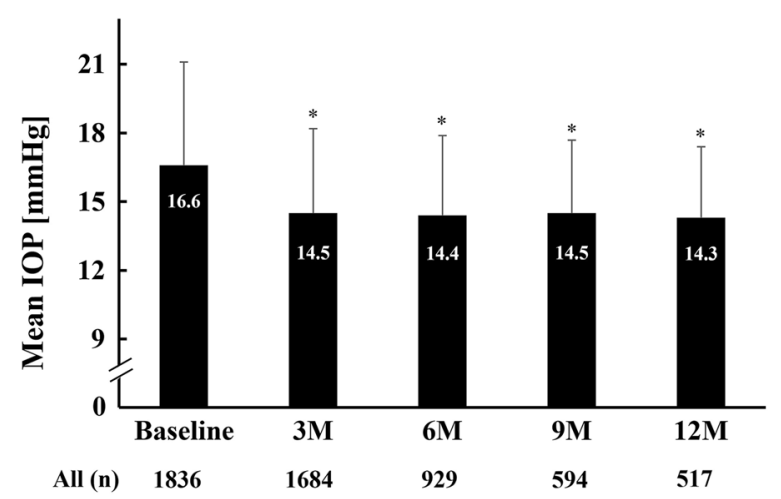

Fig. 3 Intraocular pressure (IOP) changes in all patients. The mean change in IOP for all months compared to the baseline IOP was significant $\left({ }^{*} p<0.05\right.$; paired $t$ test $)$. $n$ number of patients, $M$ months

who switched to OMDI monotherapy, the mean IOP change from the baseline to 12 months was $-0.8 \pm 2.8 \mathrm{mmHg}$ in previous drugs, such as PGAs, $-3.1 \pm 1.6 \mathrm{mmHg}$ in previous drugs such as $\beta$-blockers, and $-0.2 \pm 2.9 \mathrm{mmHg}$ in previous drugs such as fixed dose combinations of $\beta$-blockers and CAI by further sub-analysis. There was no elevation of mean IOP after switching to OMDI. This suggests that OMDI has a sufficient efficacy which can well maintain the patient's IOP which had been kept by previous glaucoma drugs even though it was PGAs.

In the concomitant therapy group, the major IOP-lowering agents added to OMDI were $\beta$ blockers or CAIs, and the use of OMDI with these agents resulted in a significant IOP reduction, suggesting its efficacy as a concomitant drug.

In this study, the use of OMDI also resulted in a significant decrease in IOP from the baseline over the 12-month observation period in patients diagnosed with NTG, POAG, or $\mathrm{OH}$.

The overall safety profile of OMDI in the current study was similar to that in the previous clinical trials $[19,22]$. The incidence of ADRs was similar among the naïve monotherapy, switching monotherapy, and concomitant therapy groups. The long-term clinical trial (RENGE) study has shown a two times increase in conjunctival hyperemia with concomitant use of OMDI with timolol [22]. In contrast, the rate of hyperemia did not increase in patients with concomitant use of timolol and other

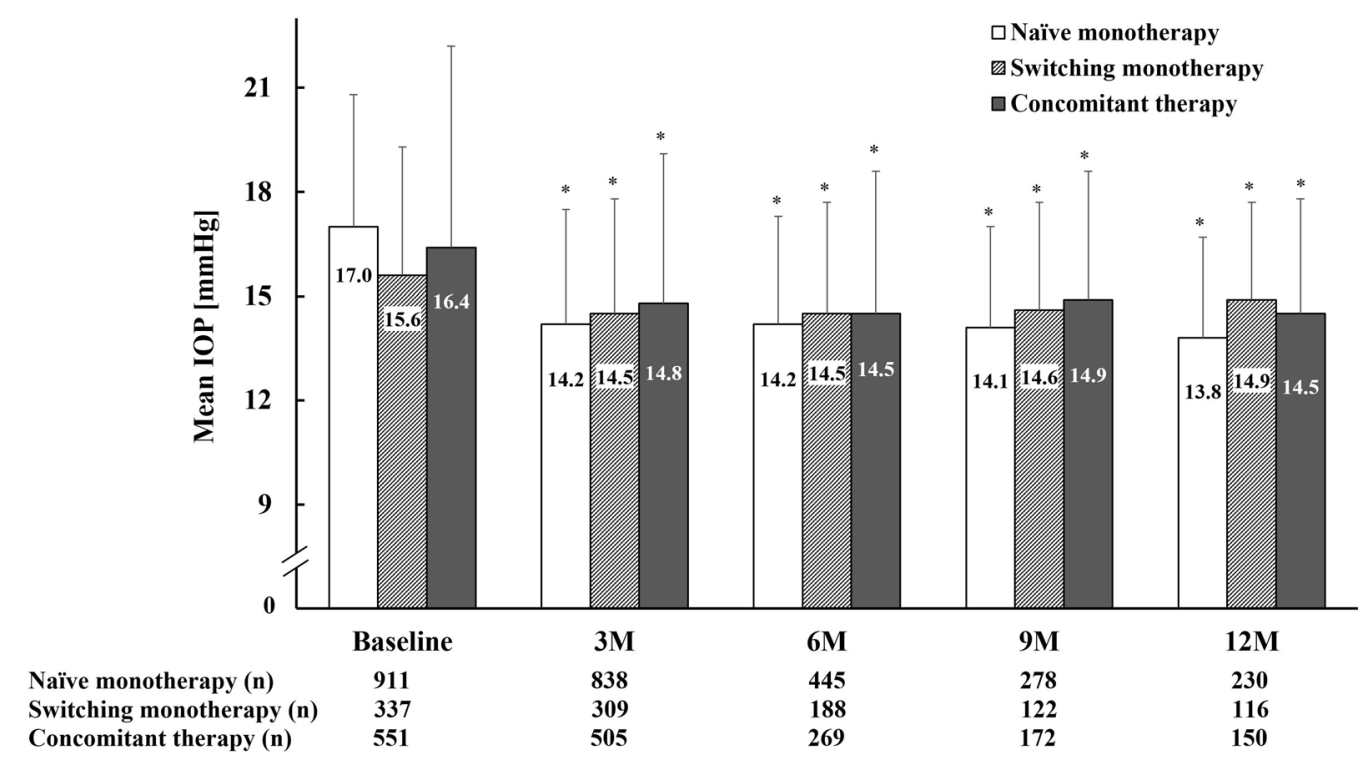

Fig. 4 IOP changes according to the therapeutic pattern. The change in mean IOP for all months compared to the baseline IOP was significant $\left({ }^{*} p<0.05\right.$; paired $t$ test $) . n$ number of patients, $M$ months 


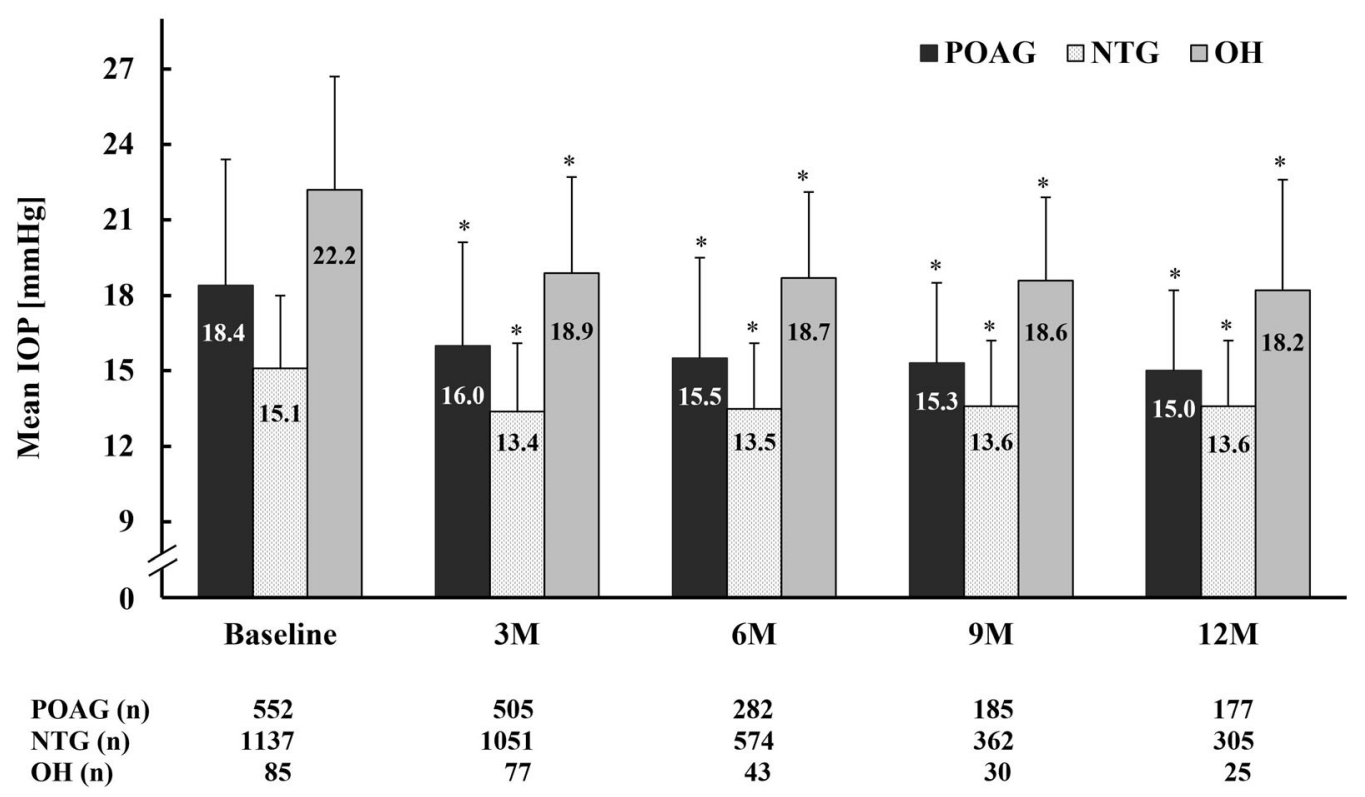

Fig. 5 IOP changes according to the diagnosis. The change in mean IOP for the different diagnoses compared to the baseline IOP was significant $\left({ }^{*} p<0.05\right)$ at all time points (paired $t$ test). $n$ number of patients, $M$ months

glaucoma ophthalmic solutions in this study. This was likely because patients adapted to incidence of hyperemia with previous antiglaucoma drugs.

In previous clinical trials, the total incidence of macular edema was $5.2 \%$ among 267 patients treated with OMDI. In addition, macular edema was observed in $26.9 \%$ of 52 patients with pseudophakic eyes and no macular edema was observed in patients with phakic eyes [24]. The incidence of macular edema in this study was substantially lower (4 cases, $0.2 \%$ ) than in previous reports, possibly because previous clinical trials enrolled pseudophakic patients, whereas this study excluded pseudophakic subjects who were contraindicated from OMDI. It is also considered that the incidence of macular edema $(0.2 \%)$ in this study was low in patients with phakic eyes is similar to previous clinical trials. Currently, the cause of the macular edema induced by OMDI is unclear. Including macular edema, the mechanism of the ocular inflammation caused by OMDI may be related to the fact that prostaglandin E2 is well known to be an inflammatory substance. The median onset date in this study was also shorter (41.5 days) than reported in a previous long-term clinical trial (240 days) [22]; the shorter onset was possibly due to the earlier diagnosis of the condition by ophthalmologists who received the safety risk communication on OMDI's ADRs. A slight increase as $13.9 \sim 14.6 \mu \mathrm{m}$ in mean central corneal thickness was observed after 12 months of treatment. Similar minor increases in central corneal thickness have been reported in clinical trials on OMDI and another EP2 agonist, taprenepag isopropyl $(<24 \mu \mathrm{m}$ for both) [25]. The variation in central corneal thickness did not affect the vision and refraction throughout the 12-month observation period; hence, the increase was not considered clinically significant.

Since antiglaucoma ophthalmic solutions are usually used for a very long time, they must have high treatment persistence rates. In this study, $82.4 \%$ of patients persistently used OMDI treatment throughout the 12 months. Nakakura et al. reported that the total persistence of OMDI at 12 months as $70 \%$ in a retrospective two-institute study [26]. Arias et al. reported that the 1-year monotherapy persistence of latanoprost, travoprost, and timolol as around 


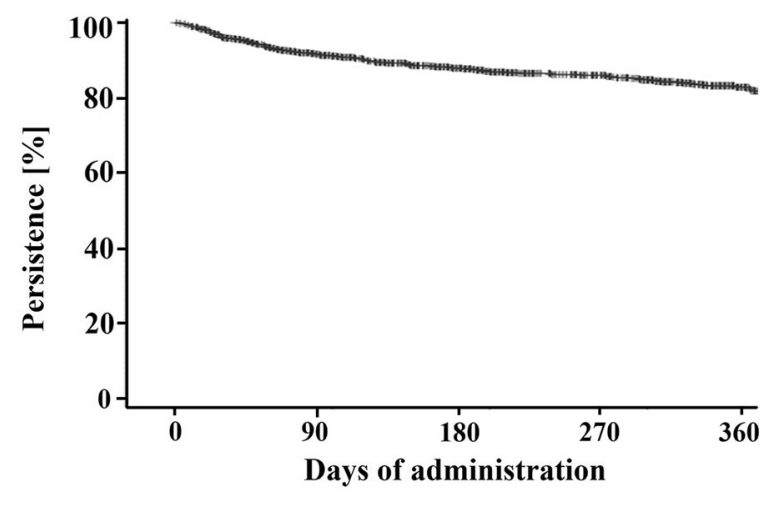

$\begin{array}{lllll}\text { (n) } 1862 & 1323 & 942 & 638 & 189\end{array}$

Fig. 6 Kaplan-Meier survivorship trajectories for treatment continuation

$90 \%, 80 \%$, and $80 \%$, respectively, in a retrospective two-institute study [27]. Kuwayama et al. reported that the 1-year persistent rate of tafluprost was $84.6 \%$ (95\% CI: $83.4-85.7 \%$ ) reported in a previous PMS study (2017). The treatment persistency in this study is considered to be comparable to the persistency of these glaucoma drugs, and suggests that OMDI is appropriate for a long-term antiglaucoma therapy [28].

In this study, there was a finding which will be a benefit for glaucoma patents that no ADR which was categorized as PAP was observed, which was consistent with the results of previous non-clinical $[29,30]$ and clinical studies [22]. OMDI was considered to be a preferable treatment option, especially for younger female patients and, indeed, this study included more number of females. In addition, there was a finding that there were limited numbers of patients who used PGAs concomitantly (38 patients, 6.6\%) in the concomitant therapy group. The background considered is that PGAs should be used concomitantly under especially careful monitoring, which is emphasized in safety risk communications, because there would be a higher risk of ocular inflammation. The detailed mechanism of the higher risk has not been known; however, there would be a PGA class effect of the contraindicated drug, tafluprost.
Overall, OMDI demonstrated a significant reduction in IOP, with no new safety concerns during this interim observation period of 12 months. It was also well tolerated in terms of both safety and efficacy; therefore, OMDI can be a new treatment option for IOP reduction in patients with glaucoma and $\mathrm{OH}$ with naïve, switching, and concomitant use.

This study has some limitations. Since this is an observational and non-interventional study conducted in daily clinical settings, the efficacy and safety measurements were not obtained at the scheduled time points. Patients with inadequate IOP reduction or ADRs may be switched to other medications; therefore, only patients with good responses or those who tolerated OMDI well could have possibly continued in the study, leading to a bias in the overall risk and benefit estimation, and persistence rate. The incidence of ADRs may be lower than the actual rate because the observation and examination of these items were voluntary, without any interventions. This might explain the lower incidence of ADRs like conjunctival hyperemia and corneal thickening in this observational study compared to that in previous clinical trials. The lower incidence of ADRs might also be due to this interim analysis, and some patients might be in their early treatment course. Hence, it cannot be ruled out that the current interim analysis result has not reflected the real-would situation completely. A long-term assessment of these events will reveal the actual incidence of these risks.

\section{CONCLUSION}

This interim analysis of OMDI PMS demonstrated that OMDI was safe and effective, without causing PAP, for the treatment of glaucoma and $\mathrm{OH}$ in daily clinical settings in Japan where OMDI was approved first in the world. Therefore, OMDI can be a first-line treatment option for glaucoma in naïve patients and as a switching option and additional option for concomitant therapy. These data are expected to contribute to other countries where OMDI will be launched as reference data for their clinical settings. 


\section{ACKNOWLEDGEMENTS}

We thank the participants included in this study. We wish to express our gratitude to the physicians at the medical institutions who cooperated in the study. Sponsorship and article processing charges for this study were funded by Santen Pharmaceutical Co., Ltd., Osaka, Japan.

Funding. Support for this project and the journal's Rapid Service and Open Access fees were funded by Santen Pharmaceutical Co., Ltd., Osaka, Japan.

Medical Writing and Editorial Assistance. Medical writing assistance, in the form of the preparation and revision of the manuscript, was supported financially by Santen Pharmaceutical Co., Ltd., Osaka, Japan and provided by Enago Life Sciences, Mumbai, India, based on a draft provided by the authors, their feedback and under their conceptual direction.

Authorship. All named authors meet the International Committee of Medical Journal Editors (ICMJE) criteria for authorship for this article, take responsibility for the integrity of the work as a whole, and have given their approval for this version to be published.

Author Contributions. Toru Nakazawa, Kanji Takahashi, Yasuaki Kuwayama, Akio Nomura and Fumiki Shimada contributed to the study design, statistical analysis and interpretation of data. All authors drafted the manuscript, revised it, and decided to submit the article for publication.

Disclosures. Toru Nakazawa has received grants and personal fees from Santen Pharmaceutical, Senju Pharmaceutical, Kowa Company, Topcon Corporation, Wakamoto Pharmaceutical, Nidek Corporation, Rohto Pharmaceutical, Alcon Japan, and Novartis. Kanji Takahashi has received grants and personal fees from Santen Pharmaceutical, Alcon Japan, Hoya, Novartis, Otsuka Pharmaceutical, Senju Pharmaceutical, Kowa Company, Bayer Yakuhin, Kyowa Kirin, Allergan Japan, Ono
Pharmaceutical and Nitto Medic. Yasuaki Kuwayama has received grants and personal fees from Otsuka Pharmaceutical, Kowa Company, Ltd., Santen Pharmaceutical, Senju Pharmaceutical, Alcon Japan, Pfizer Japan, Wakamoto Pharmaceutical Co., Ltd., AMO Japan K.K, Glaukos Japan GK, Sucampo Pharma, Novartis Pharma K.K. and Aerie Pharmaceuticals. Akio Nomura is an employee of Santen Pharmaceutical Co., Ltd. Fumiki Shimada is an employee of Santen Pharmaceutical Co., Ltd.

Compliance with Ethics Guidelines. This current ongoing post-marketing, multicenter, prospective, non-interventional, observational study is being conducted in accordance with the requirements of the regulatory authority in Japan, the Good Post-Marketing Study Practice (MHLW Ordinance No. 171; December 20, 2004). Since the research protocol was reviewed and approved by the regulatory authority in Japan before beginning the study, approval by the ethics review committees at each participating medical institution is not required.

Data Availability. Data are available from the corresponding author upon reasonable request.

Open Access. This article is licensed under a Creative Commons Attribution-NonCommercial 4.0 International License, which permits any non-commercial use, sharing, adaptation, distribution and reproduction in any medium or format, as long as you give appropriate credit to the original author(s) and the source, provide a link to the Creative Commons licence, and indicate if changes were made. The images or other third party material in this article are included in the article's Creative Commons licence, unless indicated otherwise in a credit line to the material. If material is not included in the article's Creative Commons licence and your intended use is not permitted by statutory regulation or exceeds the permitted use, you will need to obtain permission directly from the copyright holder. To view a copy of this licence, visit http:// creativecommons.org/licenses/by-nc/4.0/. 


\section{REFERENCES}

1. World Health Organization. Visual Impairment and Blindness: Fact Sheet No 282. 2014. Accessed 27 Sep 2021.

2. Tham YC, Li X, Wong TY, Quigley HA, Aung T, Cheng CY. Global prevalence of glaucoma and projections of glaucoma burden through 2040: a systematic review and meta-analysis. Ophthalmology. 2014;121:2081-90.

3. Leske MC, Connell AM, Schachat AP, Hyman L. The Barbados Eye Study: prevalence of open-angle glaucoma. Arch Ophthalmol. 1994;112:821-9.

4. Iwase A, Suzuki Y, Araie M, et al. The prevalence of primary open-angle glaucoma in Japanese: the Tajimi Study. Ophthalmology. 2004;111:1641-8.

5. Kim KE, Kim MJ, Park KH, et al. Of the Korean ES, Society O. Prevalence, awareness, and risk factors of primary open-angle glaucoma: Korea National Health and Nutrition Examination survey 2008-2011. Ophthalmology. 2016;123:532-41.

6. Collaborative Normal-Tension Glaucoma Study Group. Comparison of glaucomatous progression between untreated patients with normal-tension glaucoma and patients with therapeutically reduced intraocular pressures. Am J Ophthalmol. 1998;126:487-97.

7. Leske MC, Heijl A, Hussein M, et al. Factors for glaucoma progression and the effect of treatment: the early manifest glaucoma treatment trial. Arch Ophthalmol. 2003;121:48-56.

8. Cheema A, Chang RT, Shrivastava A, Singh K. Update on the medical treatment of primary openangle glaucoma. Asia Pac J Ophthalmol (Phila). 2016;5:51-8.

9. European Glaucoma Society. European Glaucoma Society Terminology and Guidelines for Glaucoma, 4th Edition-Chapter 3: treatment principles and options Supported by the EGS Foundation: part 1: Foreword; Introduction; Glossary; Chapter 3 Treatment principles and options. Br J Ophthalmol. 2017; Chapter 3;101:130-95.

10. Prum BE, Rosenberg LF, Gedde SJ, et al. Primary open-angle glaucoma preferred practice pattern ${ }^{\circledR}$ guidelines. Ophthalmology. 2016;123:P41-111.

11. The Japan Glaucoma Society Guidelines for Glaucoma (4rd Edition). Nippon Ganka Gakkai Zasshi. 2018;122:3-53.
12. Jonas JB, Aung T, Bourne RR, Bron AM, Ritch R, Panda-Jonas S. Glaucoma. Lancet. 2017;390: 2183-93.

13. Schmier JK, Hulme-Lowe CK, Covert DW. Adjunctive therapy patterns in glaucoma patients using prostaglandin analogs. Clin Ophthalmol. 2014;8: 1097-104.

14. Tan P, Malhotra R. Oculoplastic considerations in patients with glaucoma. Surv Ophthalmol. 2016;61: 718-25.

15. Iwamura R, Tanaka M, Okanari E, et al. Identification of a selective, non-prostanoid EP2 receptor agonist for the treatment of glaucoma: omidenepag and its prodrug omidenepag isopropyl. J Med Chem. 2018;61:6869-91.

16. Kirihara T, Taniguchi T, Yamamura K, et al. Pharmacologic characterization of omidenepag isopropyl, a novel selective EP2 receptor agonist, as an ocular hypotensive agent. Invest Ophthalmol Vis Sci. 2018;59:145-53.

17. Fuwa M, Toris CB, Fan S, et al. Effects of a novel selective EP2 receptor agonist, Omidenepag isopropyl, on aqueous humor dynamics in laser-induced ocular hypertensive monkeys. J Ocul Pharmacol Ther. 2018;34:531-7.

18. Santen Pharmaceutical Co., Ltd, UBE Industries [press release]. Santen and UBE Industries Announce Receipt of Manufacturing and Marketing Approval for Glaucoma and Ocular Hypertension Treatment EYBELIS Ophthalmic Solution 0.002\% in Japan. 2018. http://www.ube-ind.co.jp/ube/en/ news/2018/20180921_01.html. Accessed 20 May 2019

19. Aihara M, Lu F, Kawata H, Iwata A, Odani-Kawabata N, Shams NK. Omidenepag isopropyl versus latanoprost in primary open-angle glaucoma and ocular hypertension: the phase 3 AYAME study. Am J Ophthalmol. 2020;220:53-63.

20. Aihara M, Ropo A, Lu F, et al. Intraocular pressurelowering effect of omidenepag isopropyl in latanoprost non-/low-responder patients with primary open-angle glaucoma or ocular hypertension: the FUJI study. Jpn J Ophthalmol. 2020;64:398-406.

21. Fuwa M, Shimazaki A, Odani-Kawabata N, et al. Additive intraocular pressure-lowering effects of a novel selective EP2 receptor agonist, Omidenepag isopropyl, combined with existing antiglaucoma agents in conscious ocular normotensive monkeys. J Ocul Pharmacol Ther. 2021;37:223-9.

22. Aihara M, Lu F, Kawata H, Iwata A, Odani-Kawabata $\mathrm{N}$. Twelve-month efficacy and safety of omidenepag isopropyl, a selective EP2 agonist, in open-angle 
glaucoma and ocular hypertension: the RENGE study. Jpn J Ophthalmol. 2021;65:810-9.

23. Aihara M, Otani S, Kozaki J, Unoki K, Takeuchi M, Minami K, Miyata K. Long-term effect of BAK-free travoprost on ocular surface and intraocular pressure in glaucoma patients after transition from latanoprost. J Glaucoma. 2012;21:60-4.

24. Aihara M. Prostanoid receptor agonists for glaucoma treatment. Jpn J Ophthalmol. 2021;65: 581-90.

25. Schachar RA, Raber S, Courtney R, Zhang M. A phase 2, randomized, dose-response trial of taprenepag isopropyl (PF-04217329) versus latanoprost $0.005 \%$ in open-angle glaucoma and ocular hypertension. Curr Eye Res. 2011;36:809-17.

26. Nakakura S, Kanamori A, Fukuma Y, Wakabayashi S, Nagata Y, Adachi M. Evaluation of early medication persistence with omidenepag isopropyl, a topical selective prostaglandin EP2 agonist, in patients with glaucoma: a retrospective two-institute study. BMJ Open. 2021;29(11):e040301.

27. Arias A, Schargel K, Ussa F, Canut MI, Robles AY, Sánchez BM. Patient persistence with first-line antiglaucomatous monotherapy. Clin Ophthalmol. 2010;26:261-728.

28. Kuwayama Y, Hashimoto M, Kakegawa R, Nomura A, Shimada F. Prospective observational post-marketing study of tafluprost for glaucoma and ocular hypertension: effectiveness and treatment persistence. Adv Ther. 2017;34:1411-25.

29. Esaki Y, Katsuta O, Kamio H, et al. The antiglaucoma agent and EP2 receptor agonist Omidenepag does not affect eyelash growth in mice. J Ocul Pharmacol Ther. 2020;36:529-33.

30. Yamamoto $\mathrm{Y}$, Taniguchi $\mathrm{T}$, Inazumi $\mathrm{T}$, et al. Effects of the selective EP2 receptor agonist Omidenepag on adipocyte differentiation in 3T3-L1 cells. J Ocul Pharmacol Ther. 2020;36:162-9. 